\title{
Estimativa de Vazão em Redes WiFi para Suporte a Aplicações HTTP
}

\author{
Paulo Vieira, Priscila S. Barreto, Jacir L. Bordim
}

\begin{abstract}
Resumo-A adoção do padrão IEEE802.11 para redes de acesso vem ganhando cada vez mais espaço. Um exemplo desta tendência é a sua utilização em projetos como Cidades Digitais. Nestes cenários, é importante possuir mecanismos que possam mensurar não apenas o desempenho mas também as características do tráfego. Este entendimento permitirá um melhor dimensionamento do canal de forma a garantir uma vazão mínima aceitável para aplicações essenciais. Neste contexto, o presente trabalho propõe uma metodologia para estimativa de vazão em redes WiFi (IEEE $802.11 \mathrm{~b} / \mathrm{g}$ ), e com base nesta estimativa e no perfil de acesso, define o número de usuários que poderão compartilhar o canal.
\end{abstract}

Palavras-Chave-Redes 802.11, Estimativa de Vazão, Análise de Tráfego em Redes Sem Fio.

Abstract-The adoption of IEEE802.11b/g as a standard for access networks is a major trend. However, the cost to build and maintain such infra-structure is still a challenging issue. In such scenarios, it is important to have well defined mechanisms to better evaluate traffic characteristics and performance to improve network sizing in order to guarantee an adequate throughput for essential applications. This understanding can help network designers to better estimate the resources needed to provide basic services with a reasonable level of quality. This task, however, has been shown to be non-trivial. The main contribution of this work is to propose a methodology to estimate the throughput and the access pattern for a basic service in the context of the IEEE802.11b/g based networks, which may help to define the number of users that can share the channel.

Keywords-Wireless networks, IEEE 802.11, Throughput estimation and traffic analysis.

\section{INTRODUÇÃO}

A popularização e baixo custo de implantação de infraestrutura de redes sem fio, associado com o interesse de democratizar o acesso à Internet em áreas urbanas, deu origem a diversos projetos, como o Cidade Digital, ou CyberCity [7]. Projetos desta envergadura contemplam uma gama de tecnologias que, de forma integrada, fornecessem acesso à Internet entre outros serviços. De forma geral, estes projetos utilizam redes WiFi (IEEE 802.11) como rede de acesso last hop. Juntamente com o surgimento de projetos desta magnitude, envolvendo milhares de usuários com acesso sem fio, sugiram os primeiros estudos que visam mensurar não apenas o desempenho mas também as características do tráfego nesses ambientes [4], [13]. O aumento no número de usuários de redes sem fio, associado a uma demanda crescente por maior velocidade e restrições de temporização por parte dos aplicativos, requerem

P. Vieira, J. L. Bordim, P. S. Barreto, Departamento de Ciência da Computação, Universidade de Brasília, Brasília-DF, Brasil, E-mails: bordim,priscila@unb.br. Este trabalho foi parcialmente financiado pela FAP-DF e CNPq. mecanismos que permitam analisar e melhorar o desempenho das camadas envolvidas no processo de transmissão [3].

Um dos tópicos que vêm sendo pesquisados em relação à área de modelagem de tráfego de redes é a vazão de um canal de comunicação [1], [4]. Por meio da medida da capacidade efetiva que um canal de comunicação, é possível obter resultados que orientem administradores de redes na modelagem dos perfis de seus usuários. Um melhor conhecimento do limite de banda permitido por usuário permitirá um melhor dimensionamento dos recursos e um melhor provisionamento de Qualidade de Serviço (QoS) para as aplicações.

Existem, no entanto, muito desafios intrínsecos a essa análise. Um exemplo é a não uniformidade da utilização dos canais de comunicação pelos diferentes usuários. Isso dificulta a avaliação de forma mais precisa das capacidades e limites de comunicação. Entre outros fatores que afetam o mensuramento acurado da vazão podemos citar interferências, congestionamentos e colisões. Nesse sentido, vários estudos em torno da modelagem dos canais de comunicação vem sendo realizados [1], [4].

No âmbito da vazão de um canal de comunicação e perfis de usuários, a modelagem de tráfego foca-se sobre qual é a vazão mínima que teria de ser associada a esse serviço de tal forma que possam se alocar diversos usuários simultâneos sem que haja degradação da capacidade total do canal. O objetivo deste trabalho é propor uma metodologia de levantamento de perfis de aplicações com base na infra-estrutura de serviços da rede de tal forma a alcançar uma QoS aceitável. Mais especificamente, este trabalho propõe uma metodologia para estimativa de vazão em redes WiFi (IEEE $802.11 \mathrm{~b} / \mathrm{g}$ ). Com base nesta estimativa é possível definir o perfil de acesso para uma dada aplicação. Como foco de estudo, foi selecionado um serviço essencial, neste caso o protocolo HTTP (Hypertext Transfer Protocol).

O restante deste documento está estruturado da seguinte forma. Na seção II são apresentados os modelos teóricos para o cálculo de vazão máxima de um canal e a sua relação com os protocolos presentes nesse processo. Nesta mesma seção são apresentados os resultados obtidos. Na seção III, é realizado um estudo sobre o protocolo HTTP, seus requisitos e o perfil de tráfego associado com este serviço. O objetivo aqui é obter insumos que permitam caracterizar as necessidades de banda e temporização deste serviço. Como resultado final, o estudo realizado permite estimar o número máximo de usuários que podem compartilhar um canal que ofereça um serviço de acesso à Internet, mantendo os requisitos mínimos necessários para este serviço. A seção IV finaliza este trabalho. 


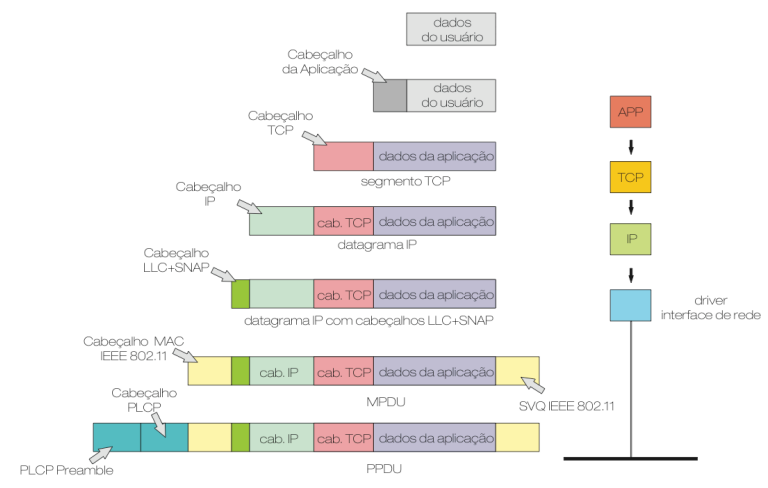

Fig. 1. Processo de encapsulamento.

\section{Estimativa de VAzÃo EM Redes WiFi}

Para que se possa calcular a vazão máxima de um dado canal deve-se supor que a rede está no seu ponto máximo de operação, ou seja o ponto de saturação. Os modelos matemáticos e estimativas apresentados neste artigo consideram um ambiente em que, nos canais de comunicação, não existem momentos de ociosidade. Neste trabalho consideramos que as transmissões realizadas na camada MAC (Medium Access Control) IEEE 802.11 utilizam o algoritmo CSMA/CA (Carrier Sense Medium Acess/Collision Avoidance) sem os mecanismos RTS/CTS.

\section{A. Considerações Iniciais}

Como pode ser observado na Fig. 1, os dados de uma dada aplicação são acoplados a um cabeçalho específico e por sua vez são repassados às camadas inferiores. Suponha que uma dada aplicação gere $n_{\text {dados }}$ bytes, já inclusos os cabeçalhos da camada de aplicação. Esses dados são repassados para a camada de transporte, a qual adiciona o cabeçalho apropriado (o TCP será considerado na discussão que segue). Aqui, assumimos um cabeçalho de 20 bytes. Na camada de rede, por sua vez, adiciona um cabeçalho de 20 bytes (protocolo IP). O datagrama é então repassado para a camada de enlace, a qual é subdividida em duas subcamadas: LLC (Logical Link Control) e MAC (medium access control). A subcamada LLC adiciona um cabeçalho (LLC+SNAP) de $3+5=8$ bytes. Na subcamada MAC é adicionado um cabeçalho de 34 bytes. Totalizando, se tem $n_{p c k}=\left(n_{\text {dados }}+20+20+8+34\right)$ bytes. Esse quadro é repassado à camada física onde são adicionados o PLCP preamble e o cabeçalho PCLP. Existem 2 tipos diferentes de preamble para realizar a sincronização entre as estações: o curto e o longo. No contexto deste trabalho, será utilizado o preamble longo (144 bits), que caracteriza uma situação pessimista. Para cada pacote TCP que é transmitido, existe um outro pacote de confirmação, ou seja, deve-se considerar duas transações em que uma, a do TCP ACK é um datagrama com $n_{\text {dados }}=0$.

Para obter-se a vazão máxima, considera-se que as informações transmitidas no canal possuem o tamanho máximo permitido. Dessa forma, considera-se que a camada IP repassa à camada de enlace um datagrama IP de 1500 bytes. Aqui são adicionados 40 bytes às informações até

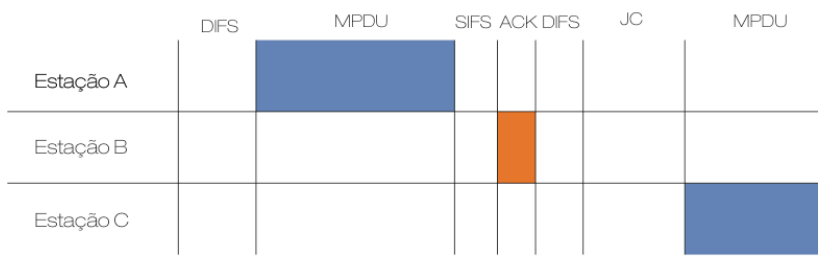

SIFS $=$ Short Inter-Frame Space

DIFS $=$ DCF Inter-Frame Space

$\mathrm{JC}=$ Contention Window

MPDU = MAC Protocol Data Unit

Fig. 2. Exemplo de Transmissão com sucesso IEEE 802.11 com CSMA/CA sem RTS/CTS.

alcançar a camada de enlace e, dessa forma, o valor máximo de $n_{\text {dados }}$ é 1460 bytes e $n_{\text {pck }}=1542$ bytes, para o pacote TCP que será transmitido. O tamanho do TCP ACK é de $n_{\text {ack }}=0+40+8+34=82$ bytes. Na camada física, a sincronização é realizada pela transmissão do PLCP Preamble, operando a 1Mbps. Ao se transmitir 144 bits esse tempo é equivalente a $144 \mu \mathrm{seg}$. O cabeçalho PLCP é transmitido a essa mesma taxa. Sendo esse cabeçalho de 48 bits a $1 \mathrm{Mbps}$ observa-se então um adicional de $48 \mu$ seg, para um total de $192 \mu$ seg ao processo de transmissão.

\section{B. Cálculo da vazão máxima: Mono-usuário}

A Fig. 2 ilustra um processo de transmissão sem RTS/CTS, que é usado como base para o cálculo da vazão máxima. Para o cálculo é necessário conhecer os valores para SIFS, DIFS e o tamanho para o ACK do IEEE 802.11. Um ACK IEEE 802.11 possui 14 bytes. O DIFS é igual a 2 vezes o tempo de slot mais SIFS. Ao considerar esses elementos, verifica-se que o tempo de uma transação é dado pela Eq. 1

$$
T_{\text {trans } 1}=D I F S+T_{\text {data }}+S I F S+T_{a c k}+T_{\text {backoff }}
$$

em que, $T_{\text {data }}$ é o tempo necessário para a transmissão dos dados do pacote, $T_{a c k}$ é o tempo para a transmissão do ACK IEEE 802.11, e $T_{\text {backoff }}$ é o tempo de backoff baseado na janela de contenção. Dessa forma, $T_{\text {data }}$ é a soma do tempo de sincronização com o tamanho dos dados divido pela taxa de transmissão do canal, conforme mostrado na Eq. 2. O tempo $T_{a c k}$ é dado pela Eq. 3 e o tempo de backoff médio é dado pela Eq. 4 , em que $J C_{\min }$ representa o tamanho da janela de contenção mínima.

$$
\begin{gathered}
T_{\text {data }}=T_{\text {plcp }}+n / \text { rate } \\
T_{\text {ack }}=T_{p l c p}+A C K / \text { rate } \\
T_{\text {backoff }}=\left(J C_{\text {min }} * t_{\text {slot }}\right) / 2
\end{gathered}
$$

Ao se substituir as Eqs. 2, 3 e 4 na Eq. 1, obtém-se a expressão para o tempo de uma transação, conforme mostrado na Eq. 5. 


$$
\begin{aligned}
T_{\text {trans } 2}= & \text { DIFS }+\frac{n_{\text {dados }}}{\text { rate }}+S I F S+\frac{A C K}{\text { rate }}+2 * T_{\text {plcp }} \\
& +\frac{J C_{\text {min }} * t_{\text {slot }}}{2}
\end{aligned}
$$

A transmissão de um pacote TCP requer duas transações, uma para o pacote e outra para o TCP ACK que será enviado. Desta forma, pode-se dizer que uma transmissão TCP $\left(T_{t c p}\right)$ é dada em função do tamanho do pacote e da taxa de transmissão (rate), as quais podem ser computadas pelas Eqs. 6 e 7. Vale ressaltar que um pacote TCP ACK possui um tamanho de 82 bytes.

$$
\begin{aligned}
T_{t c p 1}(n, \text { rate }) & =T_{\text {tpck }}(n, \text { rate })+T_{\text {tack }}(\text { rate }) \\
T_{t c p 2}(n, \text { rate }) & =2 * D I F S+2 * S I F S+4 \\
& * T_{p l c p}+J C_{\text {min }} * t_{\text {slot }}+\frac{2 * A C K}{\text { rate }} \\
& +\frac{n+82 * 8}{\text { rate }}+\frac{82 * 8}{\text { rate }}
\end{aligned}
$$

Vale ressaltar que do total de $n_{p c k}$, apenas 1460 bytes são carga útil. Dessa forma, a vazão máxima $V_{\operatorname{maxtcp} 1}$ é dada pela quantidade dos dados transmitidos no espaço de tempo $T_{t c p}$, conforme mostrado na Eq. 8. Neste trabalho considera-se que para cada pacote TCP de dados enviado é enviado um pacote de confirmação (TCP ACK), ou seja, é uma conexão interativa pura.

$$
V_{\text {Maxtcp } 1}(n, \text { rate })=(n * 8) / T_{t c p}(n * 8, \text { rate })
$$

\section{Ambientes Multi-usuários}

Quando existem diversos usuários utilizando um mesmo canal de comunicação, existe um aumento da probabilidade de colisões e de retransmissão de um mesmo pacote. Portanto, a vazão efetiva de um canal diminui. Considere que $p_{e}$ denota a probabilidade de colisão de um pacote. Neste trabalho não serão considerados erros relativos aos pacotes corrompidos. $\mathrm{O}$ tamanho médio da janela de contenção $\left(J C_{b a c k o f f}\right)$ depende diretamente das colisões, pois a cada colisão, a janela dobra seu tamanho. Esse valor é dado pela soma da multiplicação da probabilidade média do tamanho de uma janela pela possibilidade da janela realmente ter esse tamanho conforme mostrado na Eq. 9, em que $m=\log _{2}\left(J C_{\max } / J C_{\min }\right)$ [11], [13].

$$
J C_{\text {backoff }}=\frac{1-p_{e}-p_{e}\left(2 p_{e}\right)^{m}}{1-2 p_{e}} * \frac{J C_{\min }}{2}
$$

Uma estação transmite após esperar o tempo necessário para $J C_{b a c k o f f}$, $\operatorname{logo}$ colisões só ocorrerão se uma estação transmitir ao mesmo tempo que outras, isto implica que suas janelas coincidam de terminar simultaneamente. Logo, a probabilidade de uma estação $A$ colidir com $B$ é dada por $1 / J C_{\text {backoff }}$. Então, a probabilidade de colisão pode ser computada conforme Eq. 10, expressão já mostrada em [6],

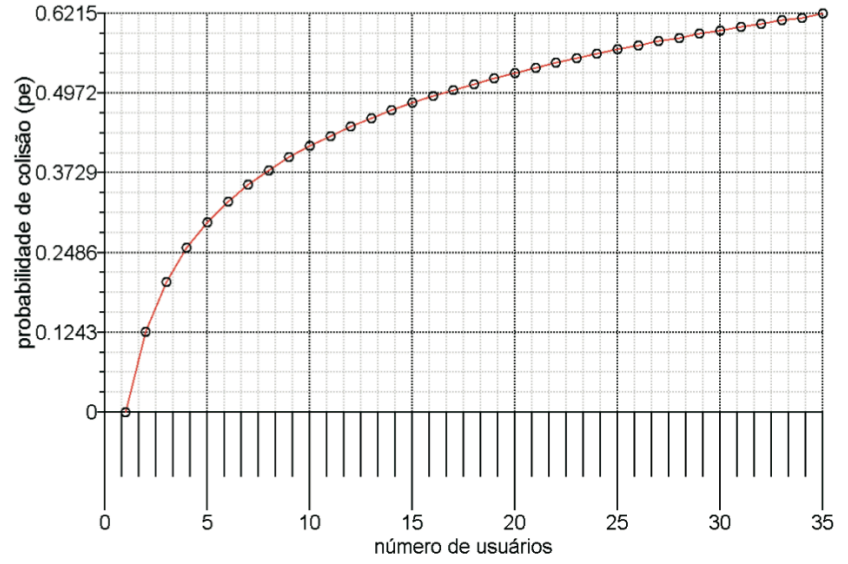

Fig. 3. Probabilidade de colisão para $p_{e}\left(J C_{\min }=32\right)$

[11], [13]. Esta equação mostra uma previsão otimista pois considera que em uma rede onde $n$ usuários se comunicam somente com pontos externos não contidos em $n$. A Fig. 3 mostra os valores da probabilidade de erros em função do número de usuários.

$$
p_{e}=1-\left(\frac{1}{J C_{\text {backoff }}}\right)^{n-1}
$$

Quando ocorre um erro na transmissão, além da alteração feita na janela de contenção, o tempo de espera para a retransmissão é $E I F S=S I F S+(8 * A C K)+T_{p l c p}+D I F S$. No entanto, como a análise se restringe aos erros como sendo apenas colisões, o EIFS não é considerado. De fato, em geral o tempo EIFS é muito pouco utilizado. Logo, o tempo de uma transmissão $\left(T_{\text {trans }}\right)$ para o caso de múltiplos usuários é dado pela Eq. 11.

$$
\begin{aligned}
T_{\text {trans } 3} & =\frac{n_{\text {dados }}}{\text { rate }}+\text { SIFS }+\frac{A C K}{\text { rate }}+\text { DIFS } \\
& +\frac{J C_{\text {min }}}{(n+1)} * t_{\text {slot }}+2 T_{\text {plcp }}
\end{aligned}
$$

Das Eqs. 10 e 11 obtém-se a expressão para a vazão que é igual a $p_{\text {Factor }} * n_{\text {dados }} / T_{\text {trans } 3}$, conforme análise mostrada em [13], em que o valor de $p_{F a c t o r}=2\left(1-p_{e}\right) /\left(2-p_{e}\right)$ engloba a probabilidade de erro no processo de transmissão.

Ao relacionar as Eqs. 10 e 11 com as Eqs. 6, 7 e 8, obtém-se a expressão mostrada na Eq. 12 para o valor de $T_{t c p 3}$.

$$
\begin{aligned}
T_{\text {tcp } 3}\left(n_{\text {dados }}, \text { rate }, n_{\text {usr }}\right) & =2 * S I F S+4 * T_{\text {plcp }} \\
& +\left(\frac{n_{\text {dados }}+82 * 8+82 * 8}{\text { rate }}\right) \\
& * \frac{2 * A C K}{\text { rate }}+2 * \text { DIFS } \\
& +2 * \frac{J C_{\text {min }}}{\left(n_{\text {usr }}+1\right)} * t_{\text {slot }}
\end{aligned}
$$

O cálculo para a vazão máxima pode ser representado conforme mostrado na Eq. 13. 


$$
\begin{array}{r}
V_{\text {Maxtcp } 2}\left(n_{\text {dados }}, \text { rate }, n_{\text {usr }}\right) \\
=P_{\text {factor }} * \frac{\left(n_{\text {dados }} * 8\right)}{T_{t c p 3}\left(n_{\text {dados }}, \text { rate }, n_{\text {usr }}\right)}
\end{array}
$$

Os valores obtidos para a vazão máxima para o protocolo TCP com base nas equações acima estão representados nas Figs. 4 e 5.

\section{Cálculo da Vazão para Redes 802.11}

O overhead fixo é intrínseco ao processo de transmissão e independe do tamanho dos dados a serem transferidos $\left(n_{\text {dados }}\right)$. O overhead fixo é composto pelos valores DIFS, SIFS, $t_{\text {slot }}, T_{p l c p}, J C_{m i n}$ e ACK. Nas redes 802.11, o tempo de envio de um símbolo é igual a $8 /$ rate, em que rate é a taxa de transmissão definida para o canal. No contexto deste artigo, é considerado 8 bits/símbolo para todas as taxas de transmissão. A Tabela 1 resume os parâmetros utilizados para as redes $802.11 \mathrm{~b}$ e $802.11 \mathrm{~g}$.

TABELA I

PARÂMETROS DOS PROTOCOLOS IEEE 802.11B E 802.11G

\begin{tabular}{|c|c|c|c|}
\hline$t_{\text {slot }}$ & MAC & DIFS & SIFS \\
\hline $20 \mu \mathrm{seg}$ & $272 \mathrm{bits}$ & $50 \mu \mathrm{seg}$ & $10 \mu \mathrm{seg}$ \\
\hline ACK & $J C_{\min }$ & $J C_{\max }$ & Taxas(Mbps) \\
\hline 112 bits & $31(802.11 \mathrm{~b})$ & 1023 & $1,2,5.5,11802.11 \mathrm{~b}$ \\
& $15(802.11 \mathrm{~g})$ & & $12,18,24,36,48,54802.11 \mathrm{~g}$ \\
\hline
\end{tabular}

Ao se substituir os valores da Tabela 1 para o cálculo de $V_{\text {maxtcp } 2}$ encontram-se os limites máximos teóricos para as diferentes taxas de transmissão. Tem-se ainda que a vazão máxima possível de um canal acontece quando os dados transmitidos possuem o tamanho máximo permitido. $\mathrm{Na}$ implementação 802.11 b, as taxas de transmissão definidas são de 1, 2, 5.5 e $11 \mathrm{Mbps}$. Esses valores permitem o cálculo do valor máximo teórico para a vazão TCP em IEEE 802.11b em função do número de usuários, conforme mostrado na Fig. 4. em que pode-se observar que para uma taxa de transmissão de 11 Mbps menos de $50 \%$ da capacidade é efetivamente utilizada na transmissão de pacotes TCP. Ainda sobre esta análise, verifica-se que para diferentes tamanhos de $n_{\text {dados }}$, a vazão máxima total se torna ainda menor. A Fig. 5 mostra a análise para a taxa de transmissão de $11 \mathrm{Mbps}$ com $n_{\text {dados }}$ igual a 256, 512, 1024 e 1460 bytes.

No padrão IEEE $802.11 \mathrm{~g}$, são adicionados, na camada física além da extensão na sincronização do sinal, 6 bits ao final de cada quadro que é enviado. Assim também, a camada física divide os dados em blocos de 216 bits. Cada símbolo demora $216 / 54 \mathrm{Mbps}=4 \mu \mathrm{seg}$ para ser enviado. Uma outra diferença é o tamanho da janela de contenção mínima, em que no padrão IEEE $802.11 \mathrm{~g}$ a tabela de contenção mínima possui tamanho 16 , com valores variando de $[0,15]$, no entanto para o padrão IEEE 802.11b este tamanho é de 32. As Figs. 6 e 7 mostram, respectivamente, a vazão máxima para diferentes taxas de transmissão e tamanho de datagramas.

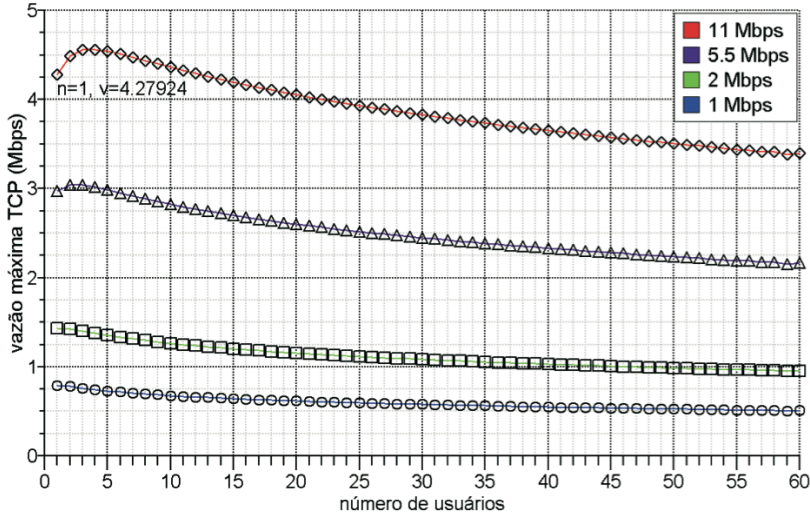

Fig. 4. Vazão Máxima TCP para diferentes taxas de transmissão em redes IEEE 802.11b.

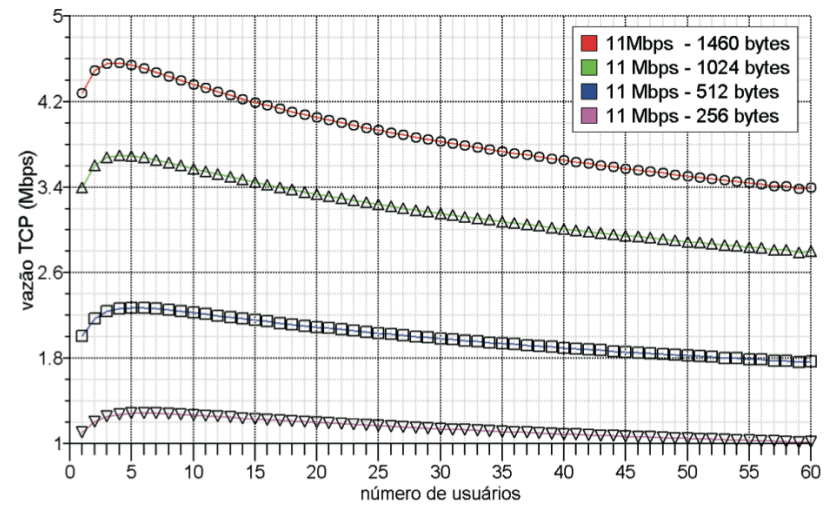

Fig. 5. Vazão TCP para diferentes tamanhos de $n_{\text {dados }}$ em redes IEEE802.11b.

\section{PERfIL DE APLiCAÇÕES}

Como discutido anteriormente, a vazão das redes IEEE $802.11 \mathrm{~b} / \mathrm{g}$ depende do número de colisões. Este fato está diretamente relacionado ao número de nós competido pelo canal e a sua periodicidade transmissão. Um outro âmbito desta análise está relacionada ao acesso destes nós e as características das aplicações que neles executam. Desta forma, identificar os limites que possibilitem executar aplicações essenciais, ou seja, respeitando os limites de temporização e Qualidade de Serviço (QoS) mínimos para sua execução, permitirá um melhor dimensionamento da infra-estrutura de rede necessária para prover tais serviços. Dentre os diversas aplicações existentes, o HTTP é um dos protocolos mais conhecidos e também um dos mais utilizados.

\section{A. Estimativa de Vazão para o Protocolo HTTP}

O HTTP é um protocolo baseado na estrutura clienteservidor. O HTTP define um conjunto de mensagens apropriadas que permitem ao cliente acessar informações (objetos) armazenados em um servidor Web. Existem dois tipos de mensagens HTTP, uma para requisições de informação por parte do cliente e uma de envio de resposta do servidor. Cada aplicação possui configurações de rede diferente que definem 


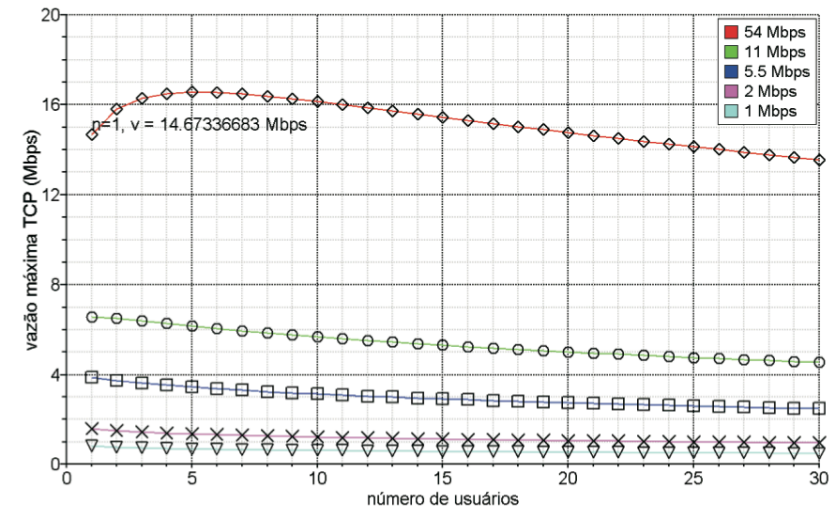

Fig. 6. Vazão Máxima TCP para diferentes taxas de transmissão em redes IEEE $802.11 \mathrm{~b} / \mathrm{g}$.

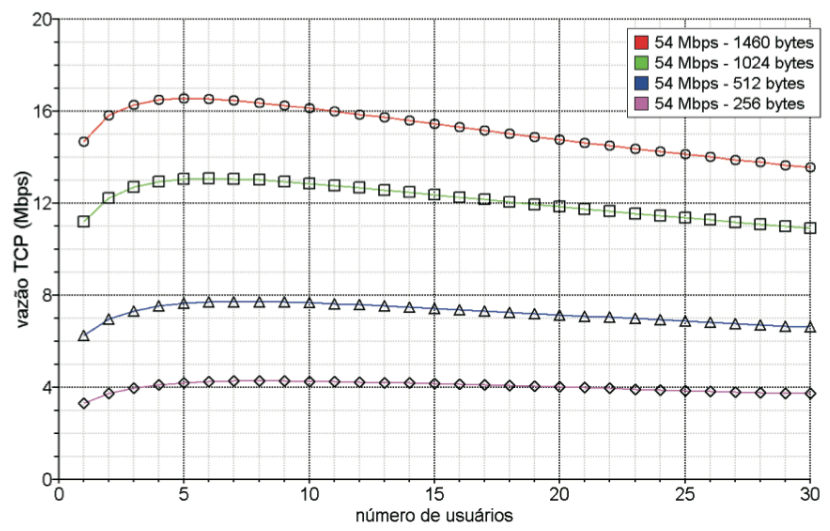

Fig. 7. Vazão TCP para diferentes tamanhos de $n_{\text {dados }}$ em redes IEEE802.11b/g.

como ela se comportará e quais os parâmetros específicos do padrão serão utilizados (e.g. HTTP 1.1 ou 1.0).

Para estimar a vazão necessária para que aplicações HTTP operem em uma rede, é necessário que se conheça o tamanho médio de uma mensagem HTTP. De acordo com [2] o tamanho médio de páginas na Internet cresceu cerca de $233 \%$ nos últimos 5 anos e o número de objetos presentes nas páginas dobraram em média. Em um estudo realizado em [9], onde foram capturados e analisados cerca de 342 milhões de pacotes, foi observado que o tamanho médio dos pacotes na Internet é de 402.7 bytes. Sendo assim, pode-se considerar que a variável $n_{\text {dados }}$ será equivalente a $n_{\text {dados }} / 402.7$ pacotes. Esses pacotes serão encapsulados e transmitidos. Para efeitos de cálculo, considera-se neste trabalho o tamanho do pacote médio igual a 512 Bytes.

Além do tamanho médio das mensagens, também é necessário conhecer o tempo médio de espera para solicitar e receber um objeto. Em [2], foi realizado um estudo, durante o período de Fevereiro de 2006 e Fevereiro de 2008, no qual verificou-se que o tempo de espera médio para as páginas Web diminuiu de 2.8seg, em 2006, para 2.33seg em 2008. Em [12] afirma-se que o tempo tolerável de espera de um usuário para a abertura de informações está em torno de 2

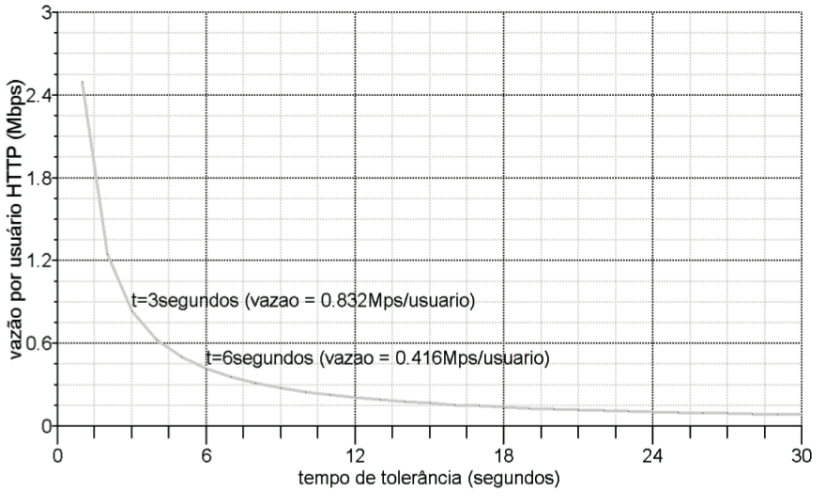

Fig. 8. Vazão HTTP para diferentes valores de $t_{\text {tolerancia }}$.

a 3 segundos, tempo este que em 2001 tinha um valor de 10 segundos aproximadamente. Vale ressaltar, no entanto, que o tempo máximo para uma transação, definido como HTTP Timeout, é atrelado à aplicação. $\mathrm{O}$ valor normalmente utilizado e recomendado é de 30 segundos [10]. A Fig. 8, mostra a vazão do protocolo HTTP, por usuário, em função do tempo de tolerância

Para que se possa calcular a vazão média necessária para que, com uma qualidade aceitável, uma aplicação HTTP possa acessar uma página de tamanho médio estimada em 312 KBytes, com um tempo médio (tolerância) de $3 \mathrm{sec}$, seria necessária uma vazão de $312 / 3=104 \mathrm{KBytes} / \mathrm{seg}$, ou 0.832 Mbps. Essa relação é mostrada na Eq. 14.

$$
V_{H T T P}\left(t_{\text {tolerancia }}\right)=\frac{\left\lceil n_{\text {HTTP }} / n_{\text {pacote }}\right\rceil}{t_{\text {tolerancia }}} * n_{\text {pacote }}
$$

\section{B. Simultaneidade de Usuários}

Em um contexto mais real, observa-se que as chances de todos os usuários $\left(n_{u s r}\right)$ estarem utilizando um mesmo canal simultaneamente são muito baixas. Portanto seria possível alocar um número maior de usuários visto que o acesso aos objetos não corresponde a um fluxo constante, mas sim a acessos esporádicos.

Foi observado que cada usuário necessita de pelo menos 104Kbps para ter um nível de qualidade aceitável para navegar na Web. A Eq. 12 permite verificar a vazão TCP para um número de usuários acessando o canal concorrentemente. Com base nestes resultados, observa-se que o número de usuários $(N U)$ que podem navegar na Web está sujeito às variáveis mostradas na Eq. 15.

$$
N U=\frac{V_{\text {MaxTCP2 }}\left(n_{\text {dados }}, \text { rate }, n_{\text {usr }}\right)}{V_{H T T P}\left(t_{\text {tolerancia }}\right)}
$$

Com base na Eq. 15, e com os resultados já obtidos na Eq. 13, tem-se $\frac{V_{M a x t c p}(512,54,9)}{104}$, que equivale a uma vazão de aproximadamente $\approx 7.5 \mathrm{Mbps}$, a qual comportaria no máximo 9 usuários simultâneos com uma conexão TCP para os parâmetros acima mostrados. No entanto, no contexto do protocolo HTTP e acesso a objetos Web, a simultaneidade de usuários está relacionada ao tempo médio 
que cada usuário estará visualizando, isto é, digerindo o objeto solicitado. Suponha-se que em um dado momento, $n_{\text {usr }}$ usuários estivessem utilizando esse serviço, porém apenas $10 \%$ do tempo. Certamente, essa ociosidade do canal poderia ser preenchida por outros usuários. De acordo com $\mathrm{o}$ instituto de pesquisas Nielsen/NetRatings juntamente com o IBOPE(Instituto Brasileiro de Opinião Pública e Estatística), o tempo médio que um usuário gasta por página da Internet é de aproximadamente 48 segundos [5], [8]. Visto de outra forma, a cada 48 segundos, o canal será utilizado durante 3 segundos e ficará 45 segundos ocioso, em média. Logo, a utilização do canal é de apenas $(3 / 48) * 100=6,25 \%$. A Eq. 16 permite computar o probabilidade de um número de usuários estarem conectados simultaneamente, dada a probabilidade de acesso em um momento específico.

$$
\operatorname{Pr}(x)=1-\sum_{j=0}^{n_{u s r}}\left(C\left(n_{u s r}, j\right) * p^{j}(1-p)^{\left(n_{u s r}-j\right)}\right.
$$

Na Eq.16, $x$ representa o números de usuários simultâneos e existe $n_{u s r}$ usuários no total compartilhando o canal. Esse valor depende também da soma de todas as probabilidades das combinações de $j \leq n_{u s r}$, em que $j$ usuários utilizam o canal ao mesmo tempo e $n_{u s r}-j$ estão ociosos. A variável $p$ corresponde à probabilidade utilização do canal e $1-p$ à probabilidade de não utilização. Assim, um administrador de rede poderia encontrar o valor de $n_{u s r}$ que se adequasse a uma margem de uma segurança e que, ao mesmo tempo, maximizasse a utilização dos recursos. Esta margem de segurança reflete a porcentagem do tempo que o canal estará disponível. Ou seja, em uma rede com $25 \%$ de margem poderia estar operando com $n_{u s r}$ usuários, onde $\operatorname{Pr}(x) \leq 75 \%$. Deve-se ressaltar que o estabelecimento de uma margem de segurança é necessária, embora a probabilidade de se extrapolar essa margem seja pequena.

Com base na Eq. 16, e com a estimativa de que cada usuário estará ativo 6,25\% do tempo, o número de usuários $\left(n_{u s r}\right)$ é $\approx 125$. Ou seja, com os parâmetros acima, um administrador de rede poderia conectar até 125 usuários em uma rede IEEE $802.11 \mathrm{~b} / \mathrm{g}$, operando a uma taxa de $54 \mathrm{Mbps}$, e disponibilizar um serviço de HTTP com uma vazão de 0.832 Mbps/usuário com uma margem de segurança de $25 \%$.

\section{CONCLusÕes}

Este trabalho propôs uma metodologia de levantamento de perfis de aplicações, com base na infra-estrutura de serviços da rede sem fio, objetivando-se alcançar uma QoS aceitável. Inicialmente, foi uma estimativa de vazão em redes WiFi (IEEE $802.11 \mathrm{~b} / \mathrm{g}$ ). Com base nesta estimativa, mecanismos para definir o perfil de acesso para uma dada aplicação foram propostos. Neste trabalho foi utilizado o protocolo HTTP (Hypertext Transfer Protocol) como base para os estudos realizados. Os resultados da análise permitem definir, com uma segurança aceitável, o número de usuários que poderão acessar o serviço mantendo-se os requisitos mínimos necessários para tal.

Vale ressaltar que os resultados obtidos são otimistas, visto que neste cálculo é considerado apenas a probabilidade de colisões entre usuários. São desconsiderados: tempo de estabelecimento de conexões, os atrasos na transmissão, as taxas de perda de pacotes, o tempo gasto com retransmissões, e o impacto da presença de interferências e ruídos. Atualmente, estudos em andamento pretendem incorporar os elementos acima mencionados no modelo inicial que propomos neste trabalho.

\section{REFERÊNCIAS}

[1] Anastasi, G.; Borgia, E.; Conti, M.; Gregori, E.; IEEE 802.11 Ad Hoc Networks: Performance Measurements, icdesw, pp.758, 23rd International Conference on Distributed Computing Systems Workshops (ICDCSW'03), 2003.

[2] Average Web Page Size Triples Since 2003. Disponível em: http://www.websiteoptimization.com/ speed/tweak/average-web-page/, último acesso: 10/05/2009.

[3] Bianchi, G. Performance Analysis of the IEEE 802.11 Distributed Coordination Function. IEEE Journal on Selected Areas in Communications, Volume 18, Issue 3, Mar 2000 Page(s):535 - 547

[4] Bruno, R.; Conti, M.; Gregori, E. Throughput Analysis and Measurements in IEEE 802.11 WLANs with TCP and UDP Traffic Flows. IEEE Transactions on Mobile Computing, Volume 7, Issue 2, Feb. 2008 Page(s): 171 .

[5] Cetic Painel IBOPE // NetRatings. Disponível em: http://www.cetic.br/usuarios/ibope/index.htm, último acesso: $10 / 05 / 2009$.

[6] Fethi Filali. Link-layer Fragmentation and Retransmission Impact on TCP Performance in 802.11-based Networks. in Proc. of MWCN 2005, 7th IFIP/IEEE International Conference on Mobile and Wireless Communications Networks, September 19th-21st 2005,Marrakech, Marrocco.

[7] Guia das Cidades Digitais. Disponível em: http://www.guiadascidadesdigitais.com.br/ site/pagina/o-que-cidadedigital, último acesso em 10/05/2009.

[8] Global Index Chart Web usage Data. Disponível em: http://www.nielsennetratings.com/resources.jsp, último acesso: 10/05/2009.

[9] NLANR - Measurement and Network Analysis. Disponível em: http://pma.nlanr.net/Datacube/, último acesso: 10/05/2009.

[10] Mozilla.org Networking Preferences. Disponível em: http://www.mozilla.org/quality/ networking/docs/netprefs.htm, último acesso: 10/05/2009.

[11] Sakurai, T.; Vu, H.L. Collision Probability in Saturated IEEE 802.11 Network,IEEE Transactions on Wireless Communications, Volume 6 , Issue 5, May 2007 Page(s):1702 - 1710.

[12] The Psychology of Web Performance. Disponível em: http://www.websiteoptimization.com/speed/tweak/ psychology-webperformance/, último acesso: 19/06/2008.

[13] Yeo, J.; and Agrawala, A.; Capacity and Variability of the IEEE 802.11 MAC Protocol. Tecnical Report No. UMIACS-TR-200345, 2003. University of Maryland, Department of Computer Science. http://www.cs.umd.edu/Library/TRs/CS-TR-4475/CS-TR-4475.ps último acesso: 10/05/2009. 